\title{
Restoring Unassisted Natural Gait to Paraplegics Via Functional Neuromuscular Stimulation: A Computer Simulation Study
}

\author{
GARY T. YAMAGUCHI AND FELIX E. ZAJAC, MEMBER, IEEE
}

\begin{abstract}
Functional neuromuscular stimulation (FNS) of paralyzed muscles has enabled spinal-cord-injured patients to regain a semblance of lower-extremity control, for example to ambulate while relying heavily on the use of walkers. Given the limitations of FNS, specifically low muscle strengths, high rates of fatigue, and a limited ability to modulate muscle excitations, it remains unclear, however, whether FNS can be developed as a practical means to control the lower extremity musculature to restore aesthetic, unsupported gait to paraplegics.

A computer simulation of FNS-assisted bipedal gait shows that it is difficult, but possible to attain undisturbed, level gait at normal speeds provided the electrically-stimulated ankle plantarflexors exhibit either near-normal strengths or are augmented by an orthosis, and at least seven muscle-groups in each leg are stimulated. A combination of dynamic programming and an open-loop, trial-and-error adjustment process was used to find a suboptimal set of discretely-varying muscle stimulation patterns needed for a 3-D, 8 degree-of-freedom dynamic model to sustain a step. An ankle-foot orthosis was found to be especially useful, as it helped to stabilize the stance leg and simplified the task of controlling the foot during swing. It is believed that the proces of simulating natural gait with this model will serve to highlight difficulties to be expected during laboratory and clinical trials.
\end{abstract}

\section{INTRODUCTION}

$\mathrm{F}^{\mathrm{s}}$ NS is an especially promising means of restoring motor control to the paralayzed limbs of spinal-cord-injured (SCI) patients. In many cases of spinal-cord injury, the nerves emanating from the spinal cord below the lesion, as well as the muscles innervated by these nerves, remain intact. Since only the communication pathways from the higher-level motor control centers to the spinal cord have been severed or blocked, FNS offers a method of controlling the distal extremities by directly applying electrical stimuli to the isolated nerves and muscles. If the microprocessor-controlled muscular excitations could be suitably coordinated, perhaps the ultimate dream of restoring unsupported, natural-speed gaits to paraplegics can be realized.

For over two decades, FNS has been applied to correct

Manuscript received September 25, 1989; revised January 10, 1990.

G. T. Yamaguchi was with the Rehabilitation R\&D Center (153), Veterans Affairs Medical Center, Palo Alto, CA 94304, and the Department of Mechanical Engineering, Stanford University, Stanford, CA 94305 . He is now with the Department of Chemical, Bio, and Materials Engineering. Arizona State University, Tempe, AZ 85287 .

F. E. Zajac is with the Rehabilitation R\&D Center (153), Veterans Affairs Medical Center, Palo Alto, CA 94304, and the Department of Me chanical Engineering, Stanford University, Stanford, CA 94305.

IEEE Log Number 9036844 footdrop during hemiplegic gait [1], [2] and to enable paraplegic gait [3]-[8]. Although virtually every application of FNS for paraplegic ambulation has relied heavily upon external, weight-supporting devices and/or extensive bracing systems, FNS-assisted gaits have been sustained for several hours each day, covering distances up to one kilometer [9]. To approach these levels of performance, typical subjects must be totally committed to clinical research programs, as months of intensive effort are required to build up muscle strenghts, to implant electrodes and leads, and to train with the stimulation systems [5], [10].

Unfortunately, a myriad of technological, physiological, and psychological barriers must be broken before FNS can hope to acheive any measure of practicality and widespread success. Some of the major difficulties are as follows:

1) The high rate of fatigue, metabolic inefficiency, and low strength of electrically-stimulated muscle [11]-[16].

2) The difficulty of controlling muscle contractions to the degree necessary for finely coordinating musculotendon force [17]-[22].

3) The high level of commitment needed from subjects for proper training and strength conditioning [10].

4) A heavy reliance on orthotics to simplify control, to aid in weight support, and to provide a measure of safety [23].

5) A lack of knowledge regarding the mechanics of muscle-tendon interaction, joint function, and the dynamics of moving, multisegmented bodies [24], [25].

These difficulties are stongly coupled together. For instance, the high fatigue rate makes control of muscle-tendon force difficult over time, necessitating a rigorous training regimen. Orthoses are needed to augment the action of the relatively weak muscles but add unwanted mass that must be supported, accelerated, and decelerated. And, if orthoses are eliminated to reduce weight, the dynamical interactions between body segments makes control more difficult because the number of degrees of freedom increases.

When reciprocal gait is attempted clincially, stimuli are typically prescribed open-loop through a trial-and-error process, usually to stabilize the leg during stance, and to elicit a flexion reflex to lift the "swing"' leg [4], [5], [7], 
[9]. Because amulation is slow, and the paraplegic's weight is primarily supported by the arms through the reliance on a walker or crutches, stability is achieved and only a crude open-loop control is needed to advance the legs. Errors in positioning and accelerating the legs need not be corrected through feedback control systems because large errors can be tolerated. Flexions and extensions of particular joints may be prescribed ad hoc without necessarily considering their dynamical effects on the movements of other joints, since it is assumed that undesirable movements can be compensated for by the arms.

We submit that a more analytical, systemwide approach to applying FNS will be needed if FNS (to control walking) is ever to progress beyond the tightly-controlled research environment. To be accepted, the FNS-assisted gait should be practical, energy-efficient, and exhibit nearnormal speed and appearance. Otherwise, as experience with prosthetic devices has shown [26], [27], a serviceable but conspicuous electrically-stimulated gait will probably find limited acceptance among potential users since the risks and effort required would continue to outweigh the potential benefits. Although much improvement in each of items 1 to 5 above will be needed before near-normal gait can be achieved routinely using FNS, we can nonetheless study the feasibility of pursing this goal through computer modeling techniques. Modeling FNSassisted walking is advantageous, in that future performance of FNS systems can be studied without the constraints imposed by our current technology.

Assuming levels of improvement in items 1 to 5 above, we have examined the feasibility of achieving unsupported, near-normal walking gait in paraplegics by identifying the major control issues, and problems that need to be solved. In doing so, a framework to guide the further development of FNS systems has been established so that paraplegics might someday be able to walk again.

\section{METHODS}

\section{A. Simplifying Assumptions}

A number of assumptions regarding the solution to many current problems in the field will be made in order to suitably limit the scope of this study to the identification of the critical musculoskeletal control problems facing the restoration of unsupported, natural gait to paraplegics.

First, we will limit the study to a specific set of conditions;

A1) Walking is considered to occur only upon smooth, level surfaces and to be completely undisturbed by external forces (e.g., wind).

This assumption is necessary because only the feasibility of restoring normal-appearing gait will be evaluated. Much work is being done to develop closed-loop systems for the control of the paralyzed upper and lower extremity, and it is expected that such systems will be employed to fine-tune coordinated movements in the presence of disturbances [17], [28]-[30].
Three assumptions concern the properties of the muscles and tendons of the paralyzed extremities:

A2) Reconditioned paralyzed musculotendons may be modeled identically to normal musculotendon actuators.

A3) The selectivity of the stimulation (of individual muscles) is assumed to be perfect.

A4) Recruitment of muscles fibers is considered to be independent of muscle length.

Assumption A2 should hold as long as severe denervation of the major lower-extremity muscles does not occur, so that the nerves, muscles, and tendons distal to the spinal lesion remain functionally intact. On the other hand, FNS systems usually stimulate muscles that have been reconditioned, which convert fast twitch muscle fibers to slow twitch fibers [14], [15]. It is further expected that the reliability of electrodes, leads, and stimulators will no longer be an issue in the future, and that their performance characteristics will approach ideal levels (assumption A3). Assumption A4 is actually now realized with neural stimulation, but not with intramuscular stimulation techniques [17].

Furthermore,

A5) Metabolic concerns are neglected.

The metabolic demands of artificially-stimulated muscular contractions differ from those of voluntary contractions. The reasons behind these differences in oxygen uptake, pulmonary ventilation, and heart rate are just beginning to be explored [11]-[13]. It is possible that the metabolic demands placed upon the body during electrically-stimulated walking may be too great unless the efficiency of FNS exercise is improved.

We further assume that:

A6) Muscle spasticity is controllable.

There are indications that FNS does not aggrevate spasticity, and may even help to reduce it [5], [31], [32]. Alternately, FNS can be used to block the undesirable action potentials causing the spastic contractions in the motor nerves themselves [33].

Finally, it is assumed that:

A7) If the duty cycles and activation levels of the muscles can be reduced, fatigue will be lessened.

A8) Muscle activations are assumed to be incrementally controllable in steps of $10 \%$ of maximum voluntary capacity (MVC).

Assumption A8 was imposed so that we could use dynamic programming to find an optimal muscle activation sequence (see below). In addition, electrode stimulation efficacy may change with time, especially over the lifetime (years) of implantable FNS systems [5], [6], [14], [21]. Thus, item A7 was imposed because the ability to precisely modulate muscle force via artificial stimulation techniques may not be available for quite some time. It was further desired that muscle activation levels be constrained to $50 \%$ or less, if possible, in accordance with item A7. This assumption is based upon experiments in which different muscles [34], or motor unit populations, if stimulated sequentially in order to reduce the active duty cycles, exhibit greater endurance [15], [35]. 
With these assumptions and since few muscles are desired to actuate walking, the specific goal of this study can now be stated. Specifically, the objective was to find a minimal set of muscles that could generate approximately normal gait trajectories without requiring either high levels of force or unduly precise control of muscle activation.

\section{B. Musculoskeletal Model}

Developing a suitable gait model involves many tradeoffs. Complex models have the potential to more closely approximate the body, but they necessarily involve more computational resources and are more difficult to analyze than simpler models. We used a 3-D linkage, having $8^{\circ}$ of freedom (DOF) to describe the segment orientations of seven rigid-body segments (feet, shanks, thighs, and trunk) that captures many of the determinants of gait [36]. Three segmental angles $\left(q_{1}\right.$ to $\left.q_{3}\right)$ specify the configuration of the stance (R-side) leg in the vertical sagittal plane, while angles $\left(q_{6}\right.$ to $\left.q_{8}\right)$ define swing-leg ( $L$-side) segment orientations [Fig. 1(a)]. Though the stance leg is constrained to move in a sagittal plane, the swing leg and trunk can also move in the frontal-plane through pelvic list [angle $q_{4}$, Fig. 1(b)]. The stance leg was constrained to sagittal plane motion to limit the total number of DOF of the model, in order to reduce the computational time in running the dynamic programming algorithm [36]. Both $q_{4}$ and the sagittal-plane angle of the trunk $q_{5}$ are defined about the stance-side hip joint; all other joints are assumed to be revolute, with 1 DOF each. Inertial parameters used to specify the musculoskeletal model are for a normal male ( $76 \mathrm{~kg}$ total mass) and are given in the Appendix.

Ligamentous constraints (and joint damping coefficients) similar to those used by Audu and Davy [37], [38] were used to limit the ranges of joint flexion and extension. Some minor modifications to their parameter set were required because the reported constants sometimes defined excessively lax joints [36].

A key element of this model is that the stance leg always remains the stance leg, and the swing leg always remains the swing leg. Only $48 \%$ of the normal gait cycle (from 14 to $62 \%$ of the gait cycle) was simulated in this analysis, as the stance-side metatarsal "joint" is assumed to be permanently hinged to the ground (Fig. 2). The motion in one half of the gait cycle is assumed to be exactly mirrored by the motion observed in the other half, at least to first order. Therefore, information about $96 \%$ of the gait cycle can be obtained. The relatively short periods during which left-toe-off, and right-toe-off, occurred were neglected so that the controls could be updated at even gait-cycle intervals of $4 \%$.

"Soft" ground-reaction constraints (see [39]) were applied to the left ("swing") leg during double support [36]. The constraints consisted of a vertical force acting at the heel, a horizontal friction force, and a torque to prevent the toes from moving below the ground surface (Fig. 3). Thus, the model could be used to study both the single-
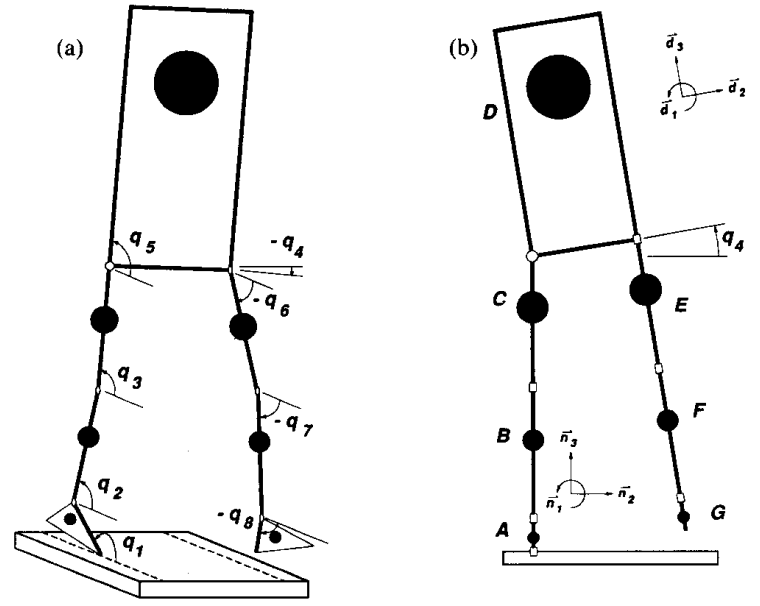

Fig. 1. (a) The 3-D, 8 DOF model, showing segment angle definitions Two degrees of freedom are evident at the stance-side hip joint $\left(q_{4}\right.$ and $q_{5}$ ); the other joints are single DOF revolute joints. (b) Front view. Note that the swing leg E-F-G remains in the plane perpendicular to basis vector $\vec{d}_{2}$.

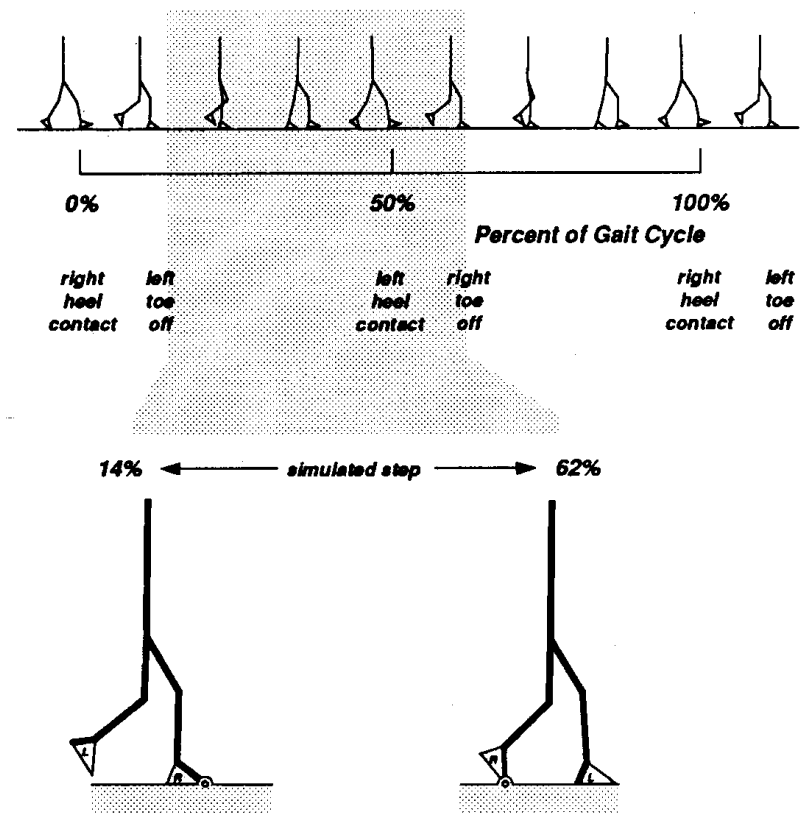

Fig. 2. The simulation period begins just after left-toe-off ( $14 \%$ of the gait cycle) and ends just before right-toe-off (62\%). Assuming bilateral symmetry, notice that $96 \%$ of the cycle is simulated.

leg stance (SLS) and double-leg support (DLS) phases of the gait cycle.

Fig. 4 depicts the Hill-type lumped-element muscle model used in series with our nonlinear tendon model to actuate the joints [24]. Activation dynamics was included for each muscle as a first-order response to a step function $u(t)$ which represented the muscle "stimulus." A time constant of $\tau=0.030 \mathrm{~s}$ [24] was utilized to approximately describe activation buildup and decay for each muscle. In accordance with assumption A8, $u(t)$ could only be set 

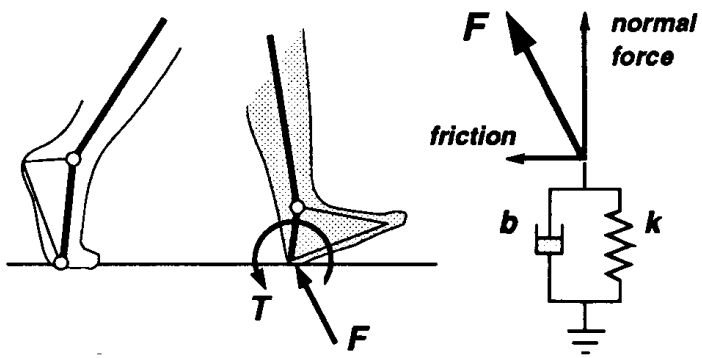

Fig. 3. "Soft" constraints acting on the left leg during double support. A normal (vertical) force, generated by a damped spring $\left(k=1.5 \times 10^{5}\right.$ $\mathrm{N} / \mathrm{m} ; b=1000 \mathrm{~N} \cdot \mathrm{s} / \mathrm{m})$, is assumed to act at the heel, causing a friction force to develop as well $(\mu \mathrm{m}=0.5) . F$ is the vector sum of these two forces. An applied torque $T$ is used, if needed, to keep the toes from violating ground surface constraints.
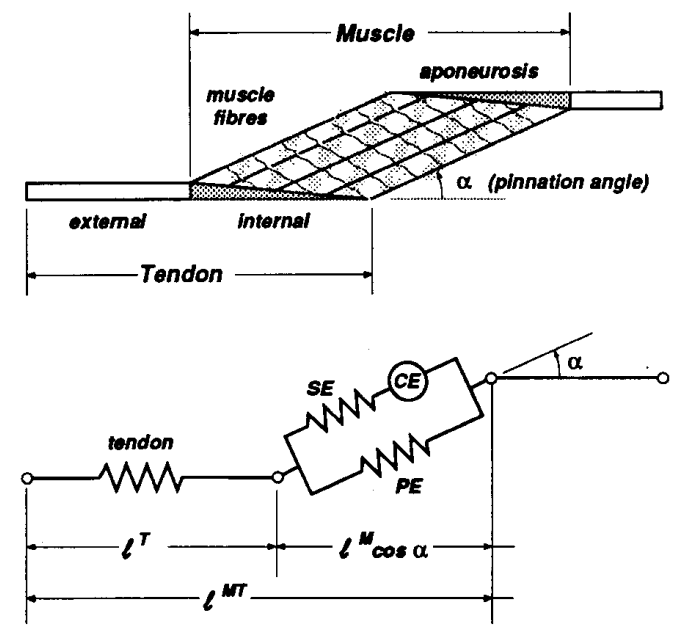

Fig. 4. Hill-type model with tendon in series. Tendon elasticity results from the tendon external to the muscle, as well as from the aponeurosis of the muscle. Muscle is assumed to be pinnated, although the angle of pinnation $(\alpha)$ changes as the muscle fibers contract. The contractile element (CE) accounts for the force-, length-, velocity property of the muscle, which also depends on the amount of activation of the muscle. A series elasticity (SE) is included to model the short-range stiffness of muscle. The passive properties of muscle are assumed to be elastic (PE). The length of the muscle fibers is $l^{M}$, and tendon length is $l^{T}$. See [24] for a review of this model.

to an integer multiple of $10 \%$. Musculotendon contraction dynamics was also included using the general relation described by Zajac et al. [40], [41],

$$
\frac{d F^{T}}{d t}=f\left(F^{T}, l^{\mathrm{MT}}, v^{\mathrm{MT}}, a(t)\right),
$$

where $F^{T}$ is the instantaneous tendon force, $l^{\mathrm{MT}}$ and $v^{\mathrm{MT}}$ are the instantaneous length and velocity of the musculotendon actuator, and $a(t)$ is the muscle activation. Thus, muscle "stimulus" $u(t)$ drives muscle activation $a(t)$ through a first-order process, and activation drives force $F^{T}(t)$ through another first-order process.

Origins, insertions, and pathways for the grouped musculotendon actuators were defined according to parameters measured by Brand et al. [42] and principles ex- plained in Hoy et al. [43]. Moments were computed about the hip and ankle joints using standard vector subtraction methods, whereas a more sophisticated model of the knee joint was used to account for the mechanical influence of the patella during knee-joint extension [44]. The same model was utilized to define moment arms for the flexors of the knee. Slight adjustments to the musculotendon parameters (tendon resting length, muscle cross section, and fiber-length) from those specified in Hoy et al. [43] were necessary to produce approximately normal isometric joint moments for the range of joint motion expected during walking. No moments were assumed at the metatarsals since it has been reported that walking can be accomplished without the toes [45].

\section{Obtaining the Suboptimal Controls}

Muscular controls were obtained through a two-phased process involving: I) coarse optimization of the controls and II) fine-tuning via trial-and-error. The first phase applied the dynamic programming algorithm of Bellman [46], [47] to determine a minimal set of musculotendon actuators and the baseline sequence of muscle activations serving to drive the state along the desired trajectory. Both muscle activation dynamics and musculotendon contraction dynamics were neglected during phase $I$ in order to reduce computational time, although the force-length and force-velocity properties of muscle were included. Once a reasonable trajectory and muscle controls (activations, in this case) were found in phase I, muscle activation and muscle-tendon contraction dynamics were reintroduced during phase II to find muscle "stimuli" $[u(t)$ in this case].

Briefly (see [36] for details), the first phase proved to be difficult as it is a multiple-input, multiple-output (MIMO) optimal control problem with a high degree of dynamic coupling among the state elements. In contrast, in phase II the "stimulus" controls could be determined simply on a muscle-by-muscle (single-input, single-output, or SISO) basis because the muscle set and their activations had already been specified in phase $\mathbf{I}$.

Phase I-Dynamic Programming: Although used mostly for problems involving few DOF, dynamic programming proved to be a workable method of solving the MIMO coordination problem for several reasons. Significantly, the algorithm computes a control solution that is dynamically optimized over the entire time interval in question, rather than being optimized quasistatically at unrelated, discrete instants of time. Another feature is that the dynamic equations of motion, i.e.,

$$
M \ddot{\vec{Q}}=\vec{T}+\vec{V}+\vec{G},
$$

do not have to be linearized. In (2), which was derived using Kane's method [48], $\boldsymbol{M}$ is the $8 \times 8$ mass matrix, $\ddot{\vec{Q}}=\left(\ddot{q}_{1}, \cdots, \ddot{q}_{8}\right)^{\prime}$ is the vector of segment angular accelerations, and $\overrightarrow{\boldsymbol{T}}, \overrightarrow{\boldsymbol{V}}$, and $\overrightarrow{\boldsymbol{G}}$ are the vectors of segmental torques applied by the muscles, coriolis and centripetal forces, and gravity, respectively. A disadvantage how- 
ever, with dynamic programming is that each of the continuous variables describing the state of the system (i.e., the $q$ 's and $\dot{q}$ 's ) must be represented, for the purposes of comparing the controls, by discrete approximations. The (bounded) controls, too, require discretization, although in our case this was readily done since we assumed the muscle activation to be incrementally variable in steps of $10 \%$.

The cost function used in the dynamic programming algorithm was initially a weighted sum of state deviations from the nominal trajectory and the sum of cubed muscle stresses, which seems to be physiologically related to muscle fatigue [49],

$$
J_{i}(k)=\sum_{l=1}^{2 n} w_{x, l}\left(x_{l}-x_{l, \mathrm{des}}\right)^{2}+\sum_{l=1}^{m} w_{u, l}\left(\frac{f_{l}}{\operatorname{PCS~\mathrm {A}_{l}}}\right)^{3}
$$

In the equation, $x_{l}$ is one of the $2 n(=16)$ elements comprising the state vector $\vec{X}=\left(\dot{q}_{1}, \cdots, \dot{q}_{8}, q_{1}, \cdots, q_{8}\right)^{\prime}$ of angular velocities and positions of the segments, $f_{l}$ and $\mathrm{PCSA}_{l}$ are the force and physiological cross-sectional area of muscle $l$, and $m$ is the number of muscles considered. The desired, gait trajectory $x_{l, \text { des }}$ was set to correspond to the nominal joint trajectories defined by Winter [50] and the foot trajectories reported by Inman et al. [51] at a walking speed of $1.36 \mathrm{~m} / \mathrm{s}$. Multiplying each state deviation by different weighting factors (i.e., the $w_{x, l}$ 's [36]) was found to be necessary since the dynamic behavior of the model was more sensitive to errors in some states than others [36]. We later found no need to minimize fatigue, and hence the cubes of muscles stresses since muscle coactivations were unlikely for a minimal set of muscles employed to effect a step. The control weighting terms (i.e., the $w_{u, i}$ 's) were accordingly set to zero for the majority of the analysis.

Since we had many degrees of freedom to control, the dynamic programming algorithm took an inordinately long time to optimize the controls. Because even slight maladjustments often resulted in disastrous "falls" in the simulated gait, a lengthy process was required to converge to a solution. This process involved: 1) selecting a set of $m$ musculotendon actuators, 2) specifying "admissible" muscle control levels at specific intervals within the step, 3) determining the optimal activation sequences through dynamic programming, 4) dynamically simulating the motions resulting from the controls, and 5) comparing the segment trajectories, ground reaction forces, and stick-figure sequences to those of normal gait. Realtime, 3-D animations of reciprocal gait constructed from the simulated stepping movements on a computer-graphics workstation illuminated subtle errors easily, and later confirmed that the steady-state stepping motions exhibited near-normal characteristics.

Steps 3 and 4 are the heart of the optimization process. First, the (discretized) dynamic programming control law was found for the predefined region of state space about the nominal (desired) state trajectory in a reverse-time process that began at the end of the simulation period ( $62 \%$ of the gait cycle) and finished at the beginning of the simulation period ( $14 \%$ gait cycle). Then, beginning with an initial system configuration, the system equations [see (2)] were integrated forward in time using the predetermined control law. Muscle activations were assumed to change instantaneously, and were updated 12 times within the step at regular "stage" intervals of $0.047 \mathrm{~s}$, based on the current system state.

For most muscles, the control algorithm chose between $0 \%$ activation and one other user-specified level that could be selected from among nine choices $(10,20, \cdots$, $90 \%$ ). Although many muscles were suitably controlled by such "on-off"' methods, the muscles of the stance leg, however, required better controls than could be afforded by "bang-bang"' controls. Otherwise, the heavily-loaded stance leg would collapse before suitable adjustments could be made in the controls at the next stage. For these muscles, the optimization routine was given a choice between two nonzero activation levels during SLS. Both the levels of activation for the $m$ musculotendon groups, and the 16 weighting values of the cost function $\left(w_{x, 1}\right)$ had to be simultaneously well-adjusted before a step could be simulated successfully [36].

Phase II-Open-Loop "Stimulus" Adjustment: Activation and the interaction of muscle and tendon primarily produce time lags in force generation [24]. Thus, the controls determined in Phase I could be used as a first guess for the "stimulus controls" in Phase II, except they had to be shifted earlier in time to account for these lags. Only a few other adjustments in the controls were then needed, and these could easily be accomplished through trial-anderror by running the simulations open-loop. Once a muscle set and "stimulus" " pattern were found that simulated normal walking, we then explored how sensitive normal gait was to the control variables. In general, gait was more sensitive to the changes in the muscle set and to "stimulus" on/off timing than to "stimulus" amplitudes.

\section{Interpreting Muscle Function}

The process of adjusting the muscle set and the admissible activation levels (Steps 1 and 2 of the dynamic programming opitimization process described above) was critically dependent upon accurately understanding the effects of each muscle on the dynamic response of the system. Here, the mechanical actions of muscles were interpreted by determining the segmental angular accelerations caused by each musculotendon component using a method suggested by Zajac and Gordon [25] (see also [51a]). Equation (2) is restructured to emphasize the dependence of the segmental accelerations upon the forces acting on the body,

$$
\ddot{\vec{Q}}=M^{-1}(\vec{T}+\vec{V}+\vec{G})
$$

The coriolis, centripetal, and gravitational force terms are zeroed by setting the angular velocities and the gravitational acceleration $(g)$ to zero, leaving the instantaneous 
vector of segmental torques applied by the muscle set $\vec{T}$. $\vec{T}$ can be decomposed into a vector sum,

$$
\overrightarrow{\boldsymbol{T}}=\sum_{j=1}^{m} \overrightarrow{\boldsymbol{T}}_{j}
$$

where $\overrightarrow{\boldsymbol{T}}_{j}$ is the vector of moments caused by a single muscle $j$. Thus, the inverse mass matrix $\boldsymbol{M}^{-1}$ works to transform $\overrightarrow{\boldsymbol{T}}_{j}$ into a vector containing the contributions to segmental angular accelerations caused by muscle $j$.

$$
\ddot{\overrightarrow{\boldsymbol{Q}}}=\boldsymbol{M}^{-1} \overrightarrow{\boldsymbol{T}}_{j} \text {. }
$$

Since $\boldsymbol{M}$ is dependent upon the physical characteristics and the instantaneous configuration of the system, the transformation is state-dependent. And because $M^{-1}$ is comprised of mostly nonzero terms, $\overrightarrow{\boldsymbol{Q}}_{j}$ will also be largely nonzero except by coincidence, implying that each individual muscle will act to accelerate every segment of the body.

For example, the segmental angular accelerations due to the soleus muscle are shown in Fig. 5. As expected, the limb segments of the right $(R)$ stance leg are accelerated the most since the right-side plantarflexors are activated. Although small, the acclerations of the left-side $(L)$ leg segments are not negligible, and therefore should be considered in analyses of muscle function. We have computed these "muscle-induced accelerations" for every configuration the model attained during the simulation [36]. Although not shown here, they form the basis for our dynamic interpretations of muscle function.

\section{REsults}

Obtaining an acceptable step motion required a delicate balance of control parameters. In the course of obtaining a step through the trial-and-error process, many hurdles to gait restoration were identified and overcome. Since these hurdles will probably have to be overcome in the clinical laboratory as well, they are discussed below.

\section{A. Critical Phases in the Simulated Gait Cycle}

Stabilizing the knee and ankle during early to mid-SLS (single-leg support, 14 to $50 \%$ of the gait cycle) was of utmost concern. If not controlled adequately, potentially disastrous falls resulted [Fig. 6(a)]. Stability was limited to a very narrow range of knee and ankle flexions, since the muscles were limited to relatively weak contractions. Even slight excesses of knee flexion or ankle dorsiflexion led to rapid, inevitable collapse since descending motions of the large heads-arm-trunk (HAT) mass were difficult to reverse.

Attaining stability of the hip in the frontal plane was of almost equal difficulty during SLS. If sufficient abduction moments were not applied in early SLS, the HAT segment would begin to list toward the swing leg under the action of gravity. In the model, recovery from falls to the side became improbable once the amount of list exceeded a few degrees.

Once the stance leg and hip were stabilized, clearance of the toe and heel during swing became the next most

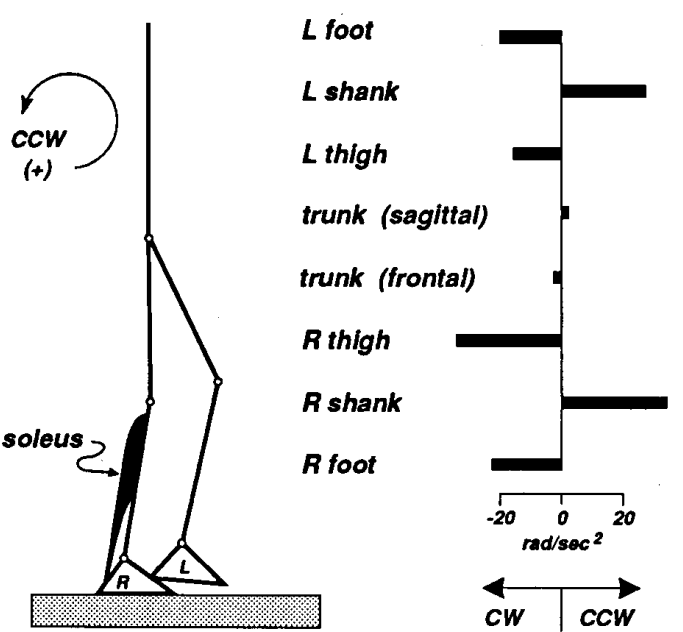

Fig. 5. Interpreting muscle function. Specifically shown here are the segmental angular accelerations (counterclockwise positive) caused by the soleus during midstance (at $34 \%$ of the gait cycle). Note that soleus accelerates all the body segments. The $R$ foot undergoes a $C W$ angular acceleration because it is not rigidly constrained. Since the angular acceleration induced in each body segment is nonzero, the gait controller must account for this dynamic coupling. Accelerations of the trunk are small because the trunk has a relatively large mass.

(a)

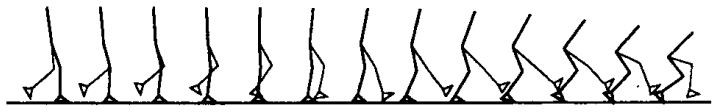

(b)

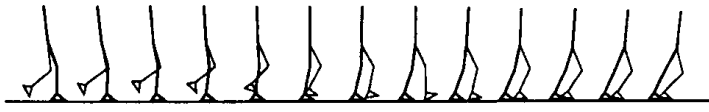

(c)

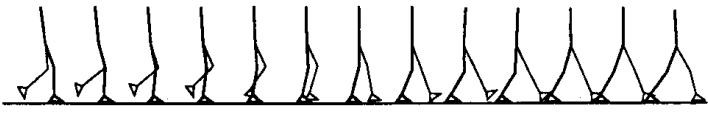

(d)

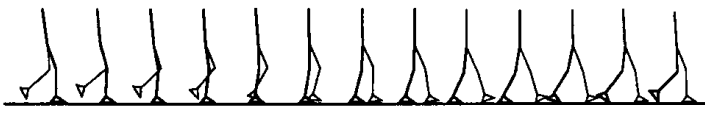

Fig. 6. Stick-figure sequences of: (a) stance leg collapse, (b) premature foot-ground contact during swing (tripping), (c) knee hyperextension in terminal swing leading to a long step, and (d) a near-normal step (bottom) obtained in spite of coarsely-controlled musculature.

important goal. Foot clearance was difficult because of the frontal-plane orientation of the pelvis and the orientation of every leg segment directly affect the height of the swing-side ankle. Additionally, the orientation of the unloaded, relatively massless foot was hard to regulate during swing using coarsely discretized control. Too much dorsiflexion or plantarflexion often resulted in premature heel or toe strikes, which usually caused the simulated walker to trip and fall [Fig. 6(b)].

Step length also needed to be carefully controlled. If knee extension during swing was too forceful, the forward momentum of the shank could easily be transferred through the hyperextended knee to the thigh, resulting in 
excess hip flexion during late SLS and a very long (in distance) step [Fig. 6(c)]. When the steps were large, it became difficult for the mass of the HAT to be transferred to the contralateral leg since the body center of mass necessarily descends more if the legs are outstretched. A large upward acceleration of the center of mass must be provided through muscle action in late SLS and DLS to propel the HAT over the supporting leg for the next step. If too short, the step appeared to mimic an unnaturally slow shuffle. The distance covered by the step therefore influenced the mechanical issues of gait, such as balance and weight transfer.

As opposed to the distance spanned by the step, the duration of the SLS phase was also important. Although controlling step duration and step length are actually separate problems, these two problems were found to be interrelated, as large steps were commonly associated with late heel strikes.

In summary, maintaining stability of the stance leg and hip during SLS, establishing clearance of the foot during swing, and controlling the step length and duration proved to be the most difficult aspects of gait to obtain. The critical stages observed during these simulations were thus between 14 and $30 \%$, near $38 \%$, and at $50 \%$ of the gait cycle, respectively.

\section{B. Muscle Actions}

Stabilizing the stance-side knee and ankle during SLS was a critically important task for soleus and vasti. Preventing collapse was accomplished through a balance of ankle plantarflexion and knee extension. With the foot flat on the floor, plantarflexor moments were essential to control the rate of ankle rotation and thus the forward progression of the shank. Doing so also acted to extend the knee, as evidenced by the CCW acceleration of the shank and the clockwise (CW) acceleration of the thigh due to soleus (Fig. 5). Both the knee extensors and the singlejoint ankle plantarflexors, then, work synergistically to restore the leg from a flexed to an upright position. This is consistent with a report by Perry [52] stating that weak knee extensors can be compensated for by normal hip extensor and calf muscles to achieve apparently normal gait.

Not only did gastrocnemius provide significant assistance to soleus during SLS, but gastrocnemius was primarily responsible for heel-rise and knee flexion during DLS just prior to toe-off. Exciting iliopsoas during late stance and DLS, because of the hip flexor moment it produces, assisted gastrocnemius in accelerating the knee into flexion in preparation for swing. Furthermore, iliopsoas stabilized the trunk in the sagittal plane by maintaining the forward momentum of the HAT.

To provide frontal-plane stability to the hip, gluteus med/minimus of the stance leg was found to provide adequate abduction moments to counteract the adduction moment imposed by gravity. For the same reason, gluteus med/minimus of the swing leg also needed to be excited just prior to heel-touch. Although gluteus maximus of the swing leg could have provided the stabilizing hip extensor moments during swing, gluteus med/minimus of the swing leg was used instead as it had a sufficient moment of force in the sagittal plane and was sure to be utilized during the following SLS phase.

To avoid striking the foot prematurely on the ground, sufficient foot-ground clearance had to be obtained near $38 \%$ of the gait cycle. Accomplishing good clearance depended upon first establishing proper ankle height above the floor, and second controlling the orientation of the foot. Minimizing segment orientation errors in nearly all the segments at $38 \%$ was essential to obtain proper ankle height. Although errors could be tolerated elsewhere in the gait cycle as long as the model did not collapse, they had to be reduced significantly by the time the ankle neared the lowest point of its trajectory. This problem was avoided in the simulations by modifying the control algorithms so that the optimization concentrated heavily upon minimizing the error at $38 \%$.

The difficulty in controlling the orientation of the foot during swing resulted from the relatively coarse nature of dorsiflexor control. Tibialis anterior and the other dorsiflexors are capable of exerting large flexor moments about the ankle, because they are used to vertically position the body while standing [53] and should be used in FNS-assisted standing as well [30], [54], [55]. When the foot is raised off the floor, however, only minute moments are required to orient its small mass relative to the shank. Since the smallest nonzero activation level available in our discretized control scheme was $10 \%$, which was too large to finely position the foot, applying any amount of dorsiflexor activity in the swing leg quickly resulted in maximal flexion. While this allowed the anterior portion of the foot to clear the ground by a large margin, it made the heel touch prematurely. Adding an externallymounted, ankle-foot orthosis (AFO) in the form of a damped, torsional spring that resists dorsiflexions past $5^{\circ}$ alleviated this problem. Though the spring was weak ( $k$ $=1.0 \mathrm{~N} \cdot \mathrm{m} / \mathrm{deg}$ ) it also aided muscle actions in the stance-leg by augmenting the plantarflexor moment when the ankle was dorsiflexed.

After these difficulties were solved, the remaining problems centered around achieving a good step length. Iliopsoas needed to be activated to achieve hip flexion since a freely-swinging ballistic motion was found inadequate. Although rectus femoris of the swing leg, if used, would have assisted iliopsoas in flexing the hip, rectus femoris was found unable to provide for the knee extension needed during swing. The reason is because its hip flexor moment not only flexes the hip, but also tends to flex the knee through dynamic coupling. This dynamic effect is strong enough to nullify the action of rectus femoris as a knee extensor. Low level activation of vasti was therefore required to extend the knee during swing, although using vasti introduced the possibility of hyperextending the knee by applying excessively strong knee extensor moments. Since vasti is strong compared to iliopsoas, only $20 \%$ activation of vasti was found to cause the hip to accelerate toward extension, even in the presence of high (50\%) ac- 
tivation of iliopsoas. The resulting knee hyperextension was often abrupt, and represents a potential area of concern to the actual implementation of a FNS system. Using hamstrings to brake both the hip and knee during late swing helped to alleviate this problem, and further limited the duration of swing by preventing the foot from "hovering" over the ground in terminal swing.

\section{Joint and Segment Trajectories}

A stick-figure sequence for a good step is shown in Fig. 6(d). This simulation was started from initial conditions corresponding to the nominal body kinematics at $14 \%$ of the gait cycle, although different initial conditions could be tolerated by the control algorithm. Though discrepancies from the normal range of joint motion exist (Fig. 7), a real-time animation of this walking movement on a computer-graphics workstation appears smooth and nearly normal. The ankle angle exhibits the greatest degree of variance, especially during late stance and DLS (40 to $62 \%$ ) when it is excessively dorsiflexed (arrow, Fig. 7). Although the plantarflexors are activated at near-maximal levels during this interval, heel-rise was difficult to initiate, which in turn exaggerated ankle dorsiflexion of this leg.

Since eversion of the stance-side ankle and lateral trunk bending [Fig. 8(b)] were not included in the model, pelvic list could not be tolerated because the abductors would be overstressed [compare Fig. 8(a) and 8(b)]. To avoid this, the pelvis had to be maintained in a near-horizontal position throughout the simulated step.

\section{Muscle Set and Activation Patterns}

As stated above, ten musculotendon groups were found necessary to produce a step. They are soleus, gastrocnemius, vasti, gluteus med/minimus, iliopsoas of the stance leg to stablize the leg during SLS and to provide initiation of swing; iliopsoas, vasti, hamstrings, and the dorsiflexors of the swing leg to control step length and foot-ground clearance; and gluteus med/minimus of the left ("swing") leg to provide stability during DLS (Fig. 9). Since gluteus med/minimus, iliopsoas, and vasti are used on both the stance and swing-leg sides, 14 muscle groups (and thus greater than 14 stimulation channels) would be required to actuate the model through a complete stride. It was found unnecessary to activate gluteus maximus since doing so (in an attempt to aid hip extension of the forward leg during DLS) tended to instead cause the trunk to fall backward. Of course, since the model exhibited only a small number of degrees-of-freedom compared to the real body, only a few muscle groups were needed to drive it along the desired state trajectory. It is felt that these do, however, represent the major components needed to maintain a steady-state stepping motion.

The resulting pattern of muscle activations shows the characteristic first-order rise and decay of activation (shaded curves, Fig. 10) to step changes in the levels of muscle stimulation (dashed lines). Although no costs were levied against activations, we were able to keep the acti-
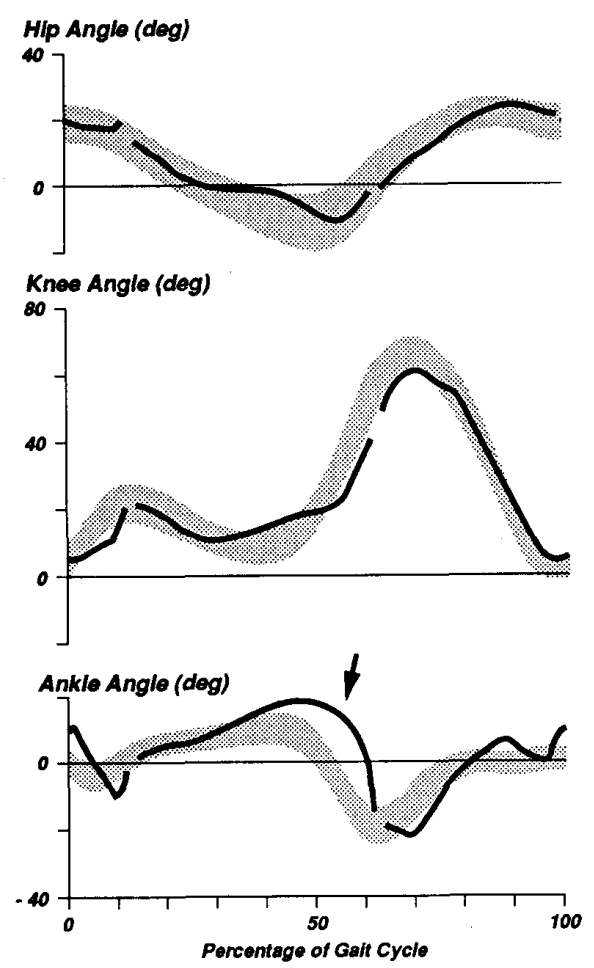

Fig. 7. Joint angles during the simulated step (black line segments) compared to the range of joint angles found in normal gait (shaded areas) [50], [51]. The curves from $0-12 \%$ and $64-100 \%$ are computed from the trajectory of the swing leg. Excessive ankle dorsiflexion is indicated by the arrow. Gaps in the simulated joint-angle curves appear because these gait-cycle epochs were not simulated.

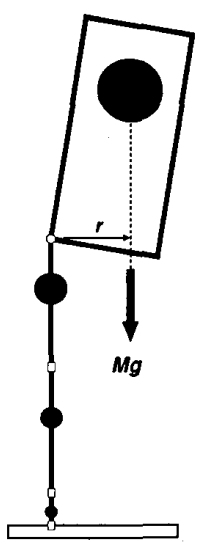

(a)

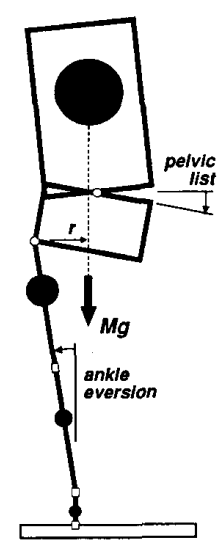

(b)
Fig. 8. (a) With a rigid (or rigidly-braced) trunk, pelvic list increases the gravity moment that must be counteracted by the abductors. (b) Lateral trunk bending reduces the gravity moment in normals, while ankle eversion allows the trunk mass to be located more nearly over the point of support. Notice that the moment arm $r$ is less when lateral trunk bending is allowed.

vation levels in the vicinity of $50 \%$ or less, except for the ankle plantarflexors. During late stance and double-leg support ( 38 to $58 \%$ of the gait cycle), near-maximal activations were needed in gastrocnemius and soleus to ef- 


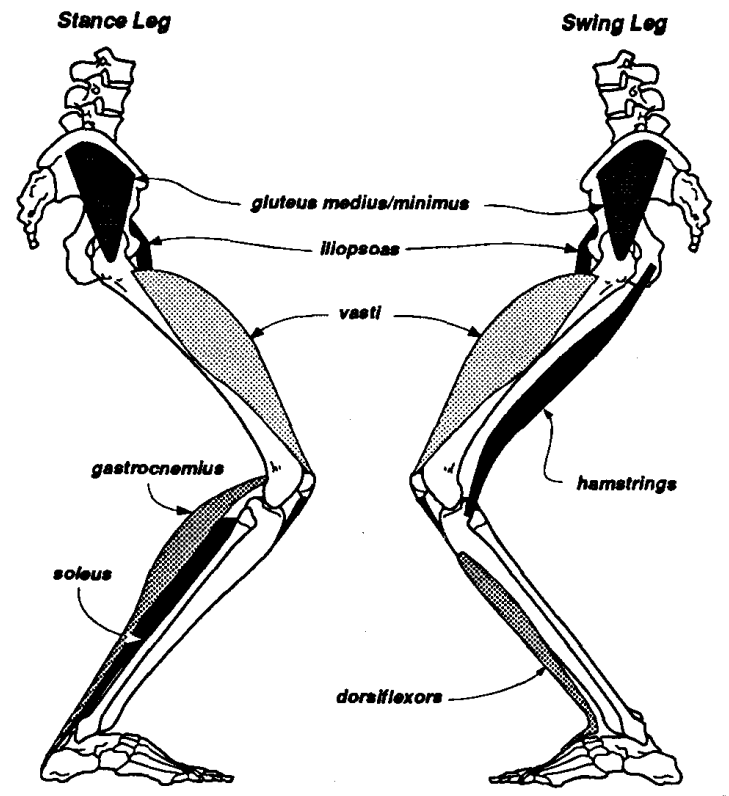

Fig. 9. The ten musculotendon actuator groups used in the step simulation. Since gluteus med/minimus, iliopsoas, and vasti of both the stance and swing-leg sides need to be controlled, only 14 stimulation channels would be required to actuate the model through a complete stride.

fect heel-rise and to generate adequate push-off forces. If constrained to activations of $50 \%$ or less, the stance-side ankle remained highly dorsiflexed throughout late SLS and DLS.

A comparison of these activations to EMG records found during gait is shown in Fig. 11. The simulated activations compare favorably to the EMG records. Activity in glutemus med/minimus during early to midstance is predicted in the model, but this activity is sustained a bit too long. Activity of iliopsoas compares well except during midswing (76-96\% of the gait cycle). Vast $i$ is used much longer during stance in the simulations, and is also used during simulated swing to extend the knee in lieu of rectus femoris. Periods of gastrocnemius, soleus, and hamstrings activation appear to match the EMG record fairly closely. The dorsiflexors are activated less often than the recordings indicate. These comparisons indicate that the means of achieving FNS-assisted gait in this simulation are similar to, but not identical with, the means actually employed by able-bodied humans. Since our goal was to find how to excite muscles to produce acceptable gait when a minimal set of muscle groups are employed, the activation patterns found would not be expected $a$ priori to mimic the natural EMG patterns. That the simulated patterns do, suggests that the CNS may also minimize the number of muscle groups recruited during walking.

\section{E. Muscle, Tendon, and Musculotendon Power}

Negative work, or work done on a musculotendon actuator by the system, results when the muscle-tendon sys- tem lengthens even though force is present in the actuator. This work may be stored as elastic energy in the tendon or absorbed by the muscle, depending on whether the lengthening occurs mostly in the tendon or in the muscle fibers themselves. The tendons here are assumed to be ideal and conservative so that any energy stored elastically is eventually released without loss.

Although tendon is considered to be conservative, the areas enclosed by the curves of tendon power (shaded areas on Fig. 12) betray that the energy stored in most of the tendons is unequal to the energy later released. This unfortunately is due to the discontinuous nature of the step simulation. When the simulation is initiated at $14 \%$, the activations of some of the muscles are assumed to be nonzero, so that force is present and energy is already stored elastically. At $62 \%$, the simulation is stopped before all the energy stored in a tendon can be released. For example, the tendon of stance-side gluteus med/minimus only releases energy, behaving as though it was stretched prior to initiating the step. Further examination of Fig. 12 reveals that the tendon of swing-side gluteus med/minimus, in contrast, only stores energy. Since the simulated movements and muscle activations are assumed to be bilaterally symmetric, the amount of energy stored just prior to stance by gluteus med/minimus is presumed to account for the amount expended just after SLS.

Bilaterally-symmetric power curves need not be assumed in order to demonstrate conservative behavior in the tendons of the ankle plantarflexors and to some extent, in the tendons of the dorsiflexors. The amount of energy stored in the elastic tendons of gastrocnemius and soleus are shown to be nearly equal to the amount expended. The 
Stance Leg
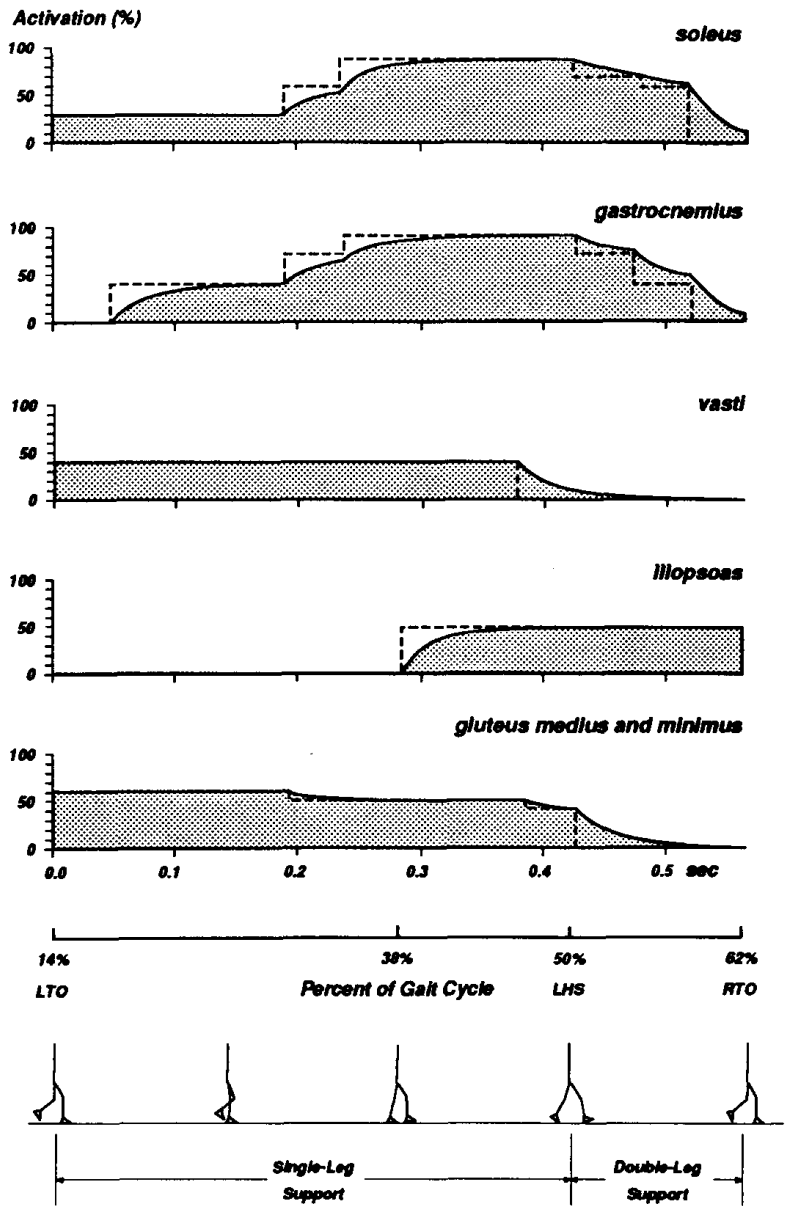

Swing Leg
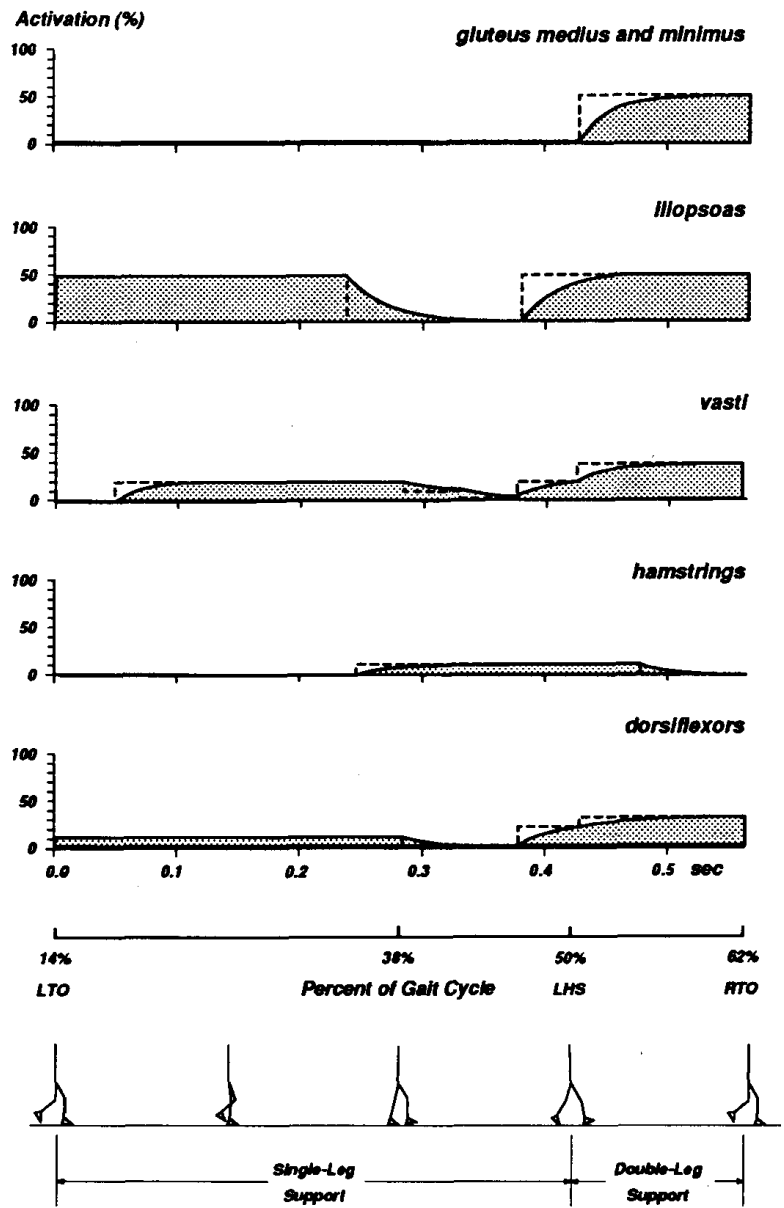

Fig. 10. Controls $u(t)$ (dashed lines) and activations $a(t)$ (shaded curves) for the ten musculotendon actuator groups during the simulated step. The plantarflexors soleus and gastrocnemius had to be highly excited during the push-off phase (38-54\% of the gait cycle). The activation of other muscles could be kept at $50 \%$ or less (except for gluteus med/minimus of the stance leg, which had to be excited somewhat more).

dorsiflexors tendon exhibits similar behavior, although the tendon is still producing power at the end of the simulation. Particularly large amounts of energy are stored in plantarflexors soleus and gastrocnemius during SLS (14$46 \%$ of the gait cycle) and released during push-off. These musculotendons actuators demonstrate classic "stretchshorten" behavior [60], [61]. Metabolic energy is conserved during stretch-shorten activity because some of the energy absorbed from the system is stored mechanically in the tendons during the initial eccentric phase, and is later expended during the second concentric phase. For instance, $78 \%$ of the peak power from soleus, and $73 \%$ of the peak power exerted by gastrocnemius during pushoff is obtained from the tendon. This is not surprising in light of the large ratios of tendon to muscle lengths for muscles crossing the ankles [24], [43].

It is also interesting to note that in mid-SLS $(30-42 \%$ of the gait cycle), the tendons of soleus and gastrocnemius absorb some of their energy (lengthen) from their muscle fibers, since their fibers expend energy (shorten) then. The same can be said for other muscles (for example, iliopsoas ) at certain periods during the gait cycle. In the case of the plantarflexors, it appears that energy from their muscle fibers and structures external to the plantarflexors is stored in the Achilles tendon at low rates over a period of time, and then released quickly during pushoff.

Power curves for hamstrings show that energy is absorbed by the muscle fibers to brake the swinging leg. Similarly, a large amount of energy is absorbed in the fibers of the dorsiflexors to prevent the forefoot from slapping the ground abruptly during DLS. Presumably, much of this energy is dissipated by the $f-v$ characteristic of the muscle fibers. 


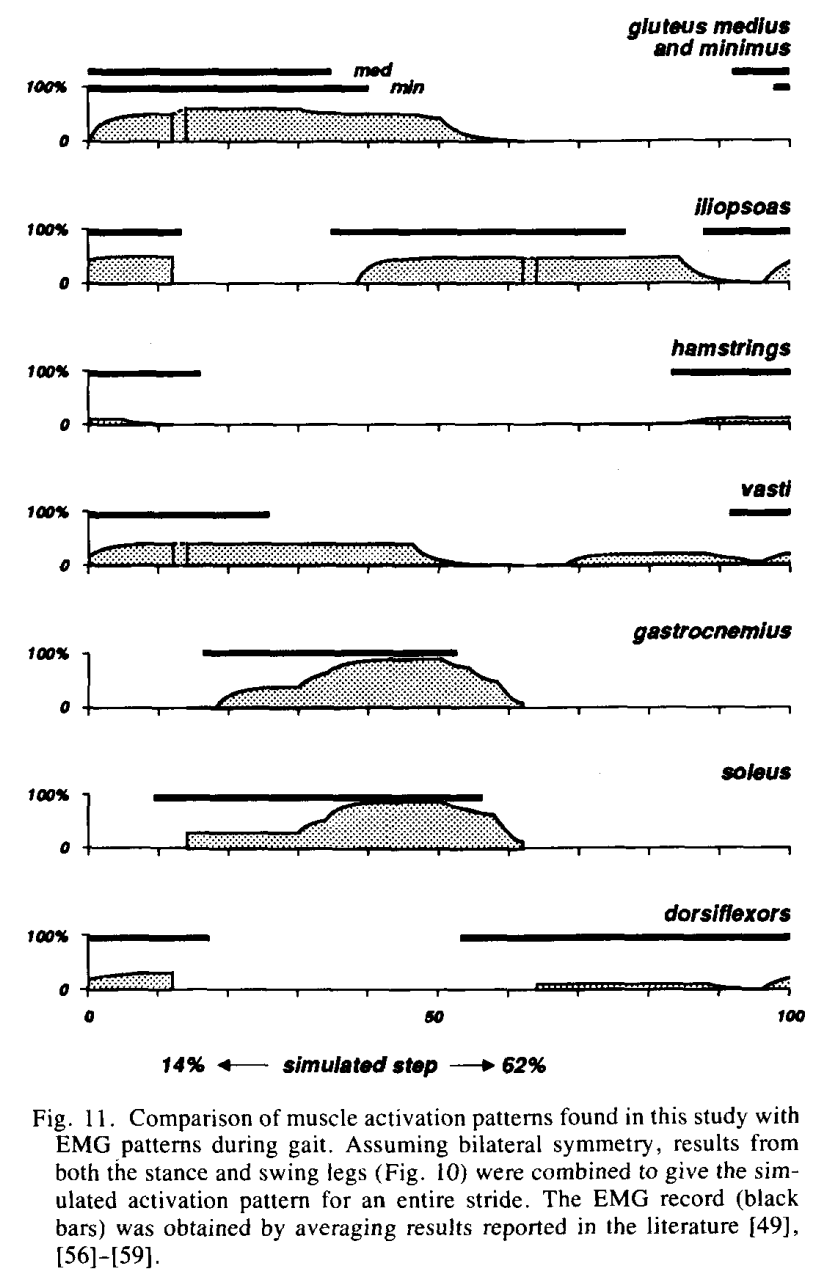

\section{F. Joint Moments}

Total joint moments at the hip, knee, and ankle for the stance leg and swing leg, which represent the combined moments due to muscle forces, ligamentous joint constraints, and the ankle-foot orthosis, indicate, not surprisingly, that the largest moments are required at the stanceleg joints (Fig. 13). We conclude that ankle plantarflexion should attain about $120 \mathrm{~N} \cdot \mathrm{m}$ (to be used during push-off) before normal-speed walking is attempted by a $76 \mathrm{~kg}$ individual. In addition, $60 \mathrm{~N} \cdot \mathrm{m}$ of hip abductor moment will be required to stabilize the stance hip in the frontal plane. With strong plantarflexors, about $54 \mathrm{~N} \cdot \mathrm{m}$ of knee extensor moment would be needed to stabilize the forward knee during DLS and the subsequent initiation of SLS.

Ankle dorsiflexion should also be strong to counteract the tendency to slap the foot on the ground during the initial weight-bearing phase of DLS. About $20 \mathrm{~N} \cdot \mathrm{m}$ was used here, although some pre-heel-strike dorsiflexor activity was prescribed between 46 and $50 \%$ to prevent footslap in the event of premature heel-strike.

\section{G. Ground Reaction Forces}

There is basic agreement between the simulated and experimental ground reaction forces (Fig. 14). The simulated vertical force resembles the familiar double-peak, although the peaks are not pronounced. It also shows that weight is transferred from one leg to the other since the left-foot force (from $0-12 \%$ ) is roughly continuous with the right-foot force ( 14 to $62 \%$ ) at left-toe-off (about $12 \%$ ). The horizontal force also exhibits the correct qualitative behavior in that decelerating forces are exerted by the ground through mid-stance, and accelerating forces are exerted from late-stance into DLS (40-60\%). The forward component of force, however, begins late (at about $42 \%$ instead of $31-36 \%$ ) and reaches only about one-half the magnitude that is recorded during normal gait.

We suspect that these deficiencies are due to the rigid, flat-bottomed nature of the foot model. For example, when foot-flat is reached in the forward leg during DLS (at about $9 \%$ ) in the simulation, the vertical and horizontal 
Stance Leg
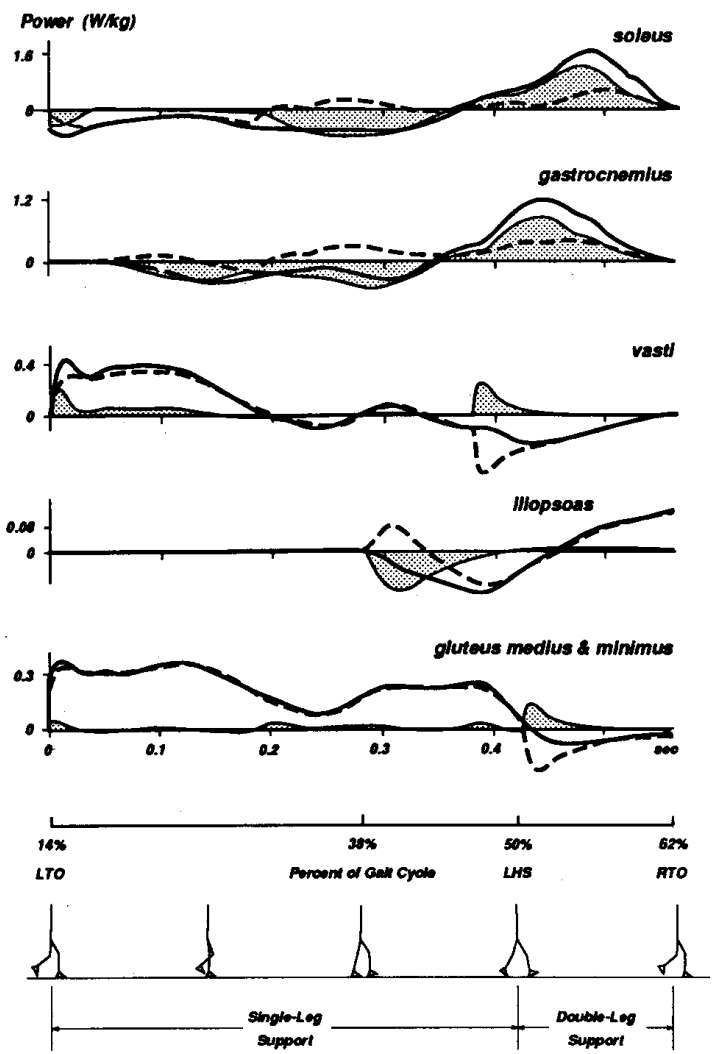

Fig. 12. Power expended (positive) and absorbed (negative) by the muscles (dashed lines), tendons (shaded curves), and musculotendon actuators (solid curves) during the simulated step. Negative power corresponds to the rate of energy absorbed by the musculotendon elements during periods of eccentric (lengthening) activity, and positive power to the rate of energy produced by the muscles and tendons during concentric (shortening) periods. The areas enclosed by the power curves and the time axis represent the energy absorbed (negative areas) or released (postive areas) by eccentric lengthening and concentric shortening periods, respectively. Values are normalized by the mass of the model $(76 \mathrm{~kg})$. ground reactions immediately increase several times in magnitude. If the point of foot-ground contact were to move forward gradually instead of so abruptly, the ground reactions should increase more smoothly. The task of finely tuning the model, its physical parameters, and the control scheme is left for further studies.

\section{Discussion}

Based on this study, it appears possible that FNS might someday enable paraplegics to walk. The prospect of walking at normal speeds is quite dubious, however, as the artificially-stimulated musculature must be quite strong (at least $50 \%$ of normal strength) in many of the muscles modeled here. In particular, the plantarflexors must be of near-normal strength to obtain heel-rise. Moments of about $120 \mathrm{~N} \cdot \mathrm{m}$ are required during push-off, which exceed the expected moment-producing capacity of the reconditioned, paralyzed muscles by $50 \%$. Other single-joint plantarflexor muscles might also be used to augment gastrocnemius and soleus, although together they would provide only about $10 \mathrm{~N} \cdot \mathrm{m}$. If gastrocnemius and soleus cannot be strengthened to levels approaching $80 \%$ of normal strength, perhaps an active device should be used to make up the $40 \mathrm{~N} \cdot \mathrm{m}$ deficit. Since an ankle-foot orthosis has already been shown here and by others [62] to be beneficial for stability, control, and safety, perhaps the powered device should take the form of a suitably designed, "power-assistive" ankle-foot, or knee-ankle-foot orthosis. Alternately, though less aesthetic, perhaps the additional push-off force can be applied to the ground by the upper body via canes or crutches.

Modeling deficiencies may accentuate these plantarflexor moment requirements somewhat, although proba- 
Stance Leg

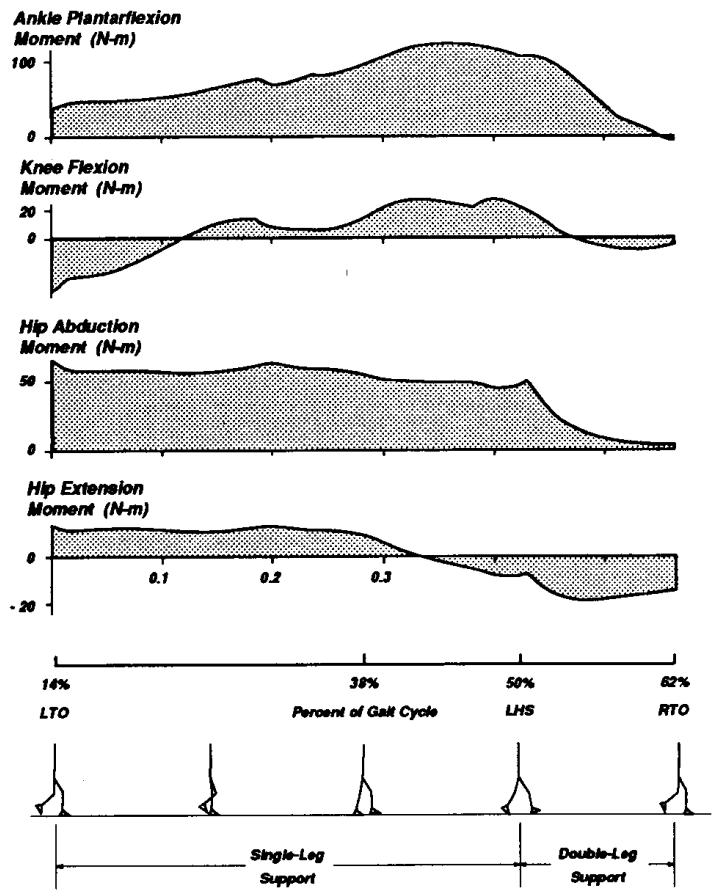

Swing Leg
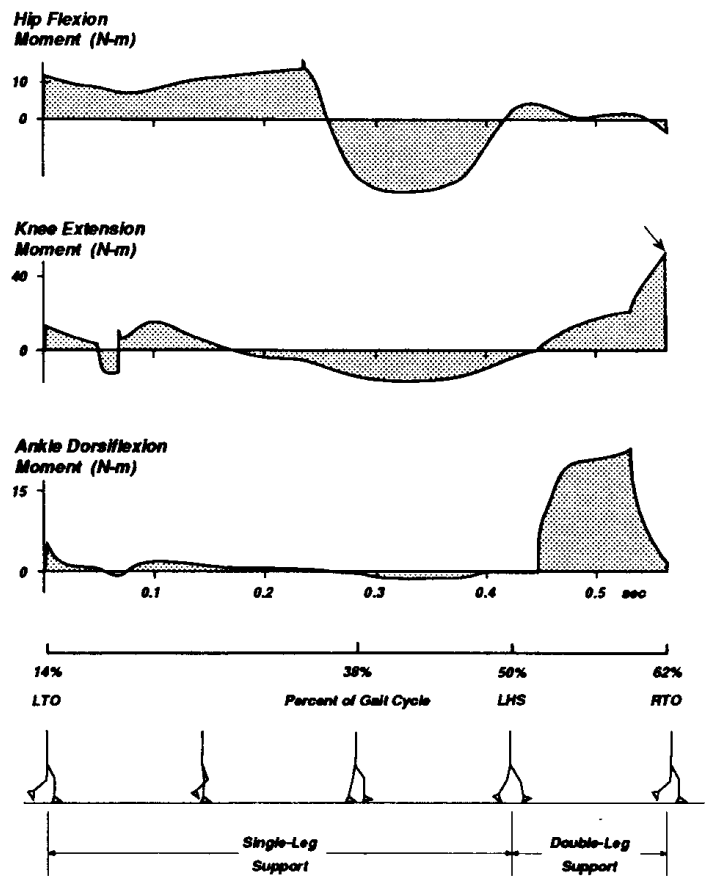

Fig. 13. Total joint moments due to muscular activity, ligamentous constraints, and the ankle AFO. The largest moments are generated at the ankle during push-off (38-54\%), and by the hip abductors during SLS A significant knee extension moment is also required in the forward leg during DLS (arrow).

bly not by $40 \mathrm{~N} \cdot \mathrm{m}$. If the moments of the plantarflexors about the toes had been included, the push-off force would have increased slightly. Also, an improved foot model with a curved plantar surface would have allowed the foot to rock forward during mid to late SLS [63], [64], and thus the heel would have been able to rise more easily. Although a curved-foot model would have reduced or eliminated the excessive amount of ankle dorsiflexion during stance, it is doubtful that the plantarflexion moments during push-off would have been reduced by much. Based on measurements by Winter [50], a peak ankle moment of $122 \mathrm{~N} \cdot \mathrm{m}$ is expected during push-off for a $76 \mathrm{~kg}$ subject walking at normal cadence. We estimate that between 72 and $89 \%$ of the normal maximum (voluntary) isometric plantarflexion capacity must be used to produce moments of these magnitudes, so the finding here that soleus and gastrocnemius were activated to levels of 80$90 \%$ MVC is reasonable.

Another factor casting doubt on the goal of unassisted paraplegic gait restoration concerns the nature of the control itself. We have shown how dynamic coupling among the moving segments complicates the control task. Indeed, because of the highly-coupled nature of the linkedsegment model, the dynamic programming method employed here proved to be arduous, and would be imprac- tical as a means of implementing closed-loop control. No matter which method is ultimately chosen to control the limbs during gait, it would have to: 1) respond quickly to disturbances, 2) remain stable in the presence of time lags, and 3) take into account the intricate dynamic interrelationships among segments. The second requirement is especially important, as lags between the arrival of the electrical stimuli and the development of substantial musculotendon force can exceed $50 \mathrm{~ms}$, which is long considering that the step period is about $560 \mathrm{~ms}$. However, we found that updating the muscle activations (but without any time lags) at stage intervals of $47 \mathrm{~ms}$ was sufficient as long as a grossly-incorrect control pattern was not applied. On the other hand, if maladjusted controls were applied to the stance leg during SLS, a single stage interval was long enough to initiate a sequence of events leading to irrecoverable collapse. Fortunately, it appears from this anaylsis that control decisions need not be exact, since rather smooth and adequately-controlled motions can result from rather crudely-modulated controls.

We suspect this to be a consequence of the nonlinear force-velocity property of the musculotendon actuators themselves. Because active force development is velocity-dependent, damping is an inherent property of the actuator [24], [65]. While the presence of damping in the 

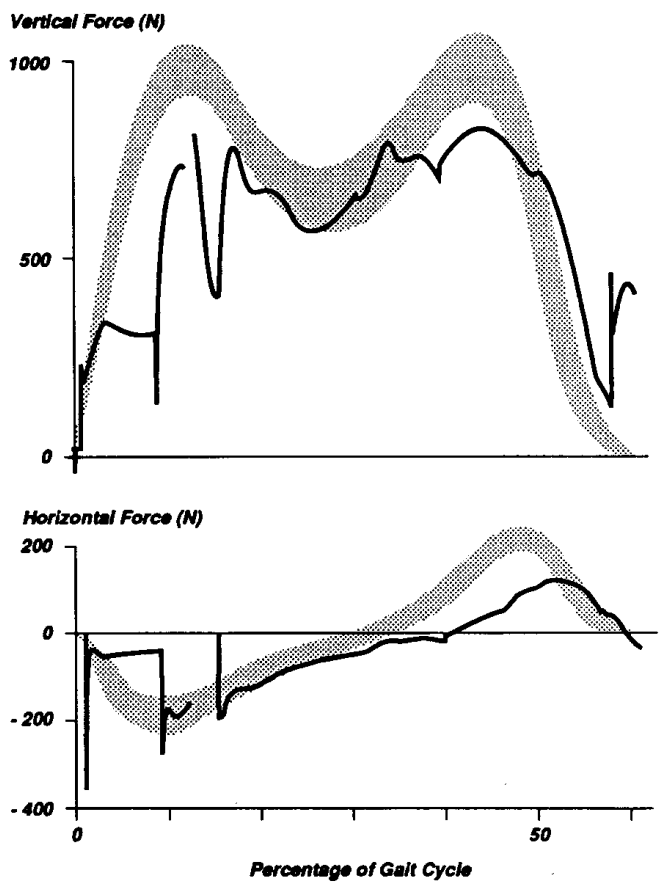

Fig. 14. Simulated vertical and horizontal ground reaction forces compared to data measured during normal gait [50]. The simulated forces (black line segments) are pieced together using the forces exerted on the left foot as the forces during DLS, and the forces exerted on the right (stance-side) foot as the forces during SLS. The simulated ground reaction forces acting on the left foot were calculated from the forces and moment acting on the swing-side foot (Fig. 3). The simulated forces acting on the right foot were calculated from the force acting at the stanceside heel and the instantaneous acceleration of the body center of mass [36]. Although the forces computed during the simulation resemble the normal gait data, the peaks are not as pronounced. Gaps in the simulated force curves appear because these gait-cycle epochs were not simulated. See text for discussion.

muscle might appear, at first, to be disadvantageous since it reduces the tension that can be developed during shortening contractions, damping works to simplify the task of controlling limb motion. In a sense, damping allows the muscle to regulate itself by: 1) exerting high moments to initiate accelerations of the segments, and 2) exerting low moments automatically once the segments begin to move and the fibers contract. Because the danger of losing control of the body segments is lessened, a simpler control scheme can be implemented.

Finally, the pattern of muscle activations needed to restore gait were found to be somewhat sensitive to body physique (i.e., inertial parameters) and to ground-foot interactions (i.e., spring and damping coefficients). However, we have not yet studied in detail the sensitivity of the controls (muscle activation pattern) to these parameters. Such studies could, for example, focus on finding the optimal shoe and ankle orthosis to exploit elastic storage in the foot-shoe-ground interface, or on the support surface inclination where gluteus maximus stimulation would become necessary (cf., "Results").

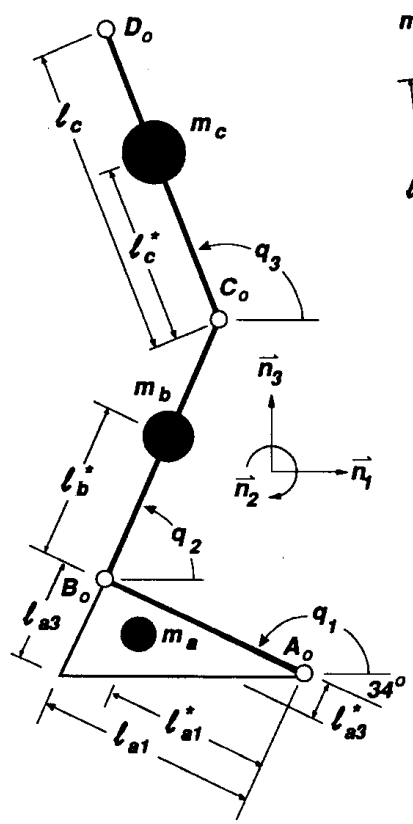

(a)

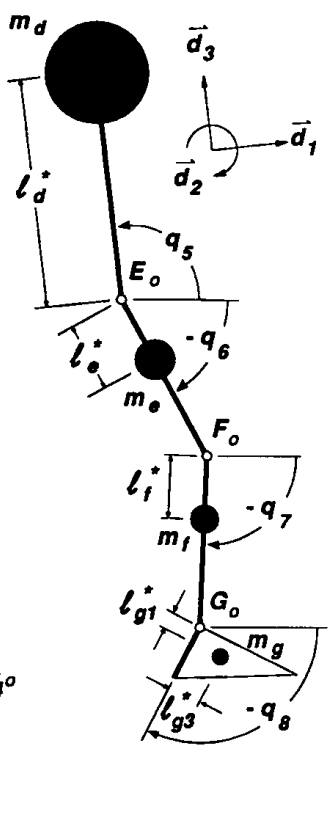

(b)
Fig. 15. Definitions of segmental parameters and axes.

\section{Conclusion}

With regard to the feasiblity of restoring normal, upright gait to paraplegics through FNS, the following conclusions based on gait simulations are made:

1) Reconditioned muscles of sufficient strength can enable a near-normal, steady-state step to be achieved. Unfortunately, the minimum levels of strength needed, specifically in the ankle plantarflexors during push-off, far exceed strengths expected with FNS. Additional push-off forces might have to be obtained via actively-powered orthotic devices, or from canes or crutches, to sustain level walking. Even a passive ankle-foot orthosis would be helpful since it would supply an additional plantarflexion moment during stance, and would simplify control of the unloaded foot during swing.

2) Control of gait must be performed on a systemwide (i.e., global) basis as the body segments are dynamically coupled together. Gait quality is highly dependent upon appropriate selection of the musculotendon actuator set and the on-off patterns of stimulation. The simulated step was less sensitive to alterations in the activation levels during periods of stimulation.

3) Every available muscle-tendon group does not need to be conditioned or stimulated to support undisturbed, level gait. The number of musculotendon groups needed depends upon the number of degrees of freedom allowed during the simulation or gait trial.

4) Activation patterns obtained using the dynamic programming optimization method were in most cases, sim- 
TABLE I

Segment Dimensions and Inertial Parameters

\begin{tabular}{|c|c|c|c|}
\hline \multicolumn{4}{|c|}{ Segment Dimensions and Inertial Parameters } \\
\hline & $\begin{array}{l}\text { lengths } \\
\text { (m) }\end{array}$ & $\begin{array}{c}\text { mass } \\
(\mathrm{kg})\end{array}$ & $\begin{array}{l}\text { principal moments } \\
\text { of inertia }\left(\mathrm{kg}-\mathrm{m}^{2}\right)\end{array}$ \\
\hline foot & $\begin{array}{l}l_{a 1}=0.175 \\
l_{a 3}=0.118 \\
l_{a 1}^{*}=0.100 \\
l_{a 3}^{*}=0.0295\end{array}$ & $m_{2}=1.10$ & $\begin{array}{l}I_{a 1}^{*}=0.002 \\
I_{a 2}^{*}=0.008 \\
I_{a 3}^{*}=0.009\end{array}$ \\
\hline shank & $\begin{array}{l}l_{b}=0.435 \\
l_{b}^{*}=0.247\end{array}$ & $m_{b}=3.75$ & $\begin{array}{l}I_{b 1}^{*}=0.019 \\
I_{b 2}^{*}=0.065 \\
I_{b 3}^{*}=0.065\end{array}$ \\
\hline thigh & $\begin{array}{l}l_{c}=0.410 \\
l_{c}^{*}=0.227\end{array}$ & $m_{c}=7.58$ & $\begin{array}{l}I_{c 1}^{*}=0.080 \\
I_{c 2}^{*}=0.126 \\
I_{c 3}^{*}=0.126\end{array}$ \\
\hline trunk & $\begin{array}{l}\ell_{d}=0.172 \\
\ell_{d}^{*}=0.343\end{array}$ & $m_{d}=51.22$ & $\begin{aligned} I_{d 1}^{*} & =0.764 \\
I_{d 2}^{*} & =3.407 \\
I_{d 3}^{*} & =3.297\end{aligned}$ \\
\hline . & $\begin{aligned} l_{a} & =l_{c} \\
l_{b}^{*} & =l_{c}-l_{c}^{*} \\
l_{f} & =l_{b} \\
l_{f}^{*} & =l_{b}-l_{b}^{*} \\
l_{g} & =l_{a 3} \\
l_{g 1}^{*} & =l_{a 3}^{*} \\
l_{g 3}^{*} & =l_{a 1}-l_{a 1}^{*}\end{aligned}$ & $\begin{array}{l}m_{0}=m_{c} \\
m_{f}=m_{b} \\
m_{g}=m_{a}\end{array}$ & $\begin{aligned} I_{\theta 1, \theta 2, \theta 3}^{*} & =I_{c 1, c 2, c 3}^{*} \\
I_{t 1, f 2, b 3}^{*} & =I_{b 1, b 2, b 3}^{*} \\
I_{g 1}^{*} & =I_{a j}^{*} \\
I_{g 2}^{*} & =I_{a 2}^{*} \\
I_{g 3}^{*} & =I_{a t}^{*}\end{aligned}$ \\
\hline
\end{tabular}

ilar in timing to reported EMG records. Care must be exercised if normal EMG patterns are used as a template for prescribing FNS stimulus patterns, however, since each active muscle acts to accelerate every body segment.

\section{APPENDIX}

Segment dimensions and axes of the 8 DOF model are defined in Fig. 15. A sagittal-plane view of the stance leg is shown in (a), while a side-view of the trunk and swing leg is depicted in (b). Note that the basis vectors of figures (a) and (b) differ because of pelvic list angle $q_{4}$ (see Fig. 1). Inertial body-segmental parameters are given in Table I.

\section{ACKNOWLEDGMENT}

The authors wish to acknowledge the support of the Rehabilitation R\&D Service, Department of Veterans Affairs. We are also grateful to Dr. M. G. Hoy and to E. L. Topp for their contributions to the musculoskeletal mod- eling portion of this study. Dr. I. Perkash provided many clinically-relevant suggestions for which we are indebted. Special thanks are also given to David (Idd) Delp for his assistance with the figures. This work is based on a Ph.D. dissertation [36] submitted by G. Y. to the Mechanical Engineering Department, Stanford University, in Aug. 1989.

\section{REFERENCES}

[1] W. T. Liberson, H. J. Holmquest, D. Scot, and M. Dow, “Functional electrotherapy: Stimulation of the peroneal nerve synchronized with the swing phase of the gait of hemiplegic patients, "Arch. Phys. Med. Rehab., vol. 42, pp. 101-105, Feb. 1961.

[2] R. L. Waters, D. McNeal, and J. Perry, "Experimental correction of footdrop by electrical stimulation of the peroneal nerve," J. Bone Joint Surg., vol. 57-A, pp. 1047-1054, Dec. 1975.

[3] D. Graupe, K. H. Kohn, and S. P. Basseas, "Control of electrically-stimulated walking of paraplegics via above- and below-lesion EMG signature identification," IEEE Trans. Automat. Cont., vol. 34 , no. 2, pp. 130-138, Feb. 1989.

[4] E. Isakov, J. Mizrahi, and T. Najenson, "Biomechanical and physiological evaluation of FES-activated paraplegic patients," $J$. Rehab. Res. Dev., vol. 23, no. 3, pp. 9-19, July 1986. 
[5] A. R. Kralj and T. Bajd, Functional Electrical Stimulation: Standing and Walking after Spinal Cord Injury. Boca Raton, FL: CRC Inc., 1989.

[6] E. B. Marsolais and R. Kobetic. "Implantation techniques and experience with percutaneous intramuscular electrodes in the lower extremities," J. Rehab. Res. Dev., vol. 23, no. 3, pp. 1-8, July 1986.

[7] J. Mizrahi, Z. Braun, T. Najenson, and D. Graupe, "Quantitative weightbearing and gait evaluation of paraplegics using functional electrical stimulation," Med. Biol. Eng. Comput., vol. 23, pp. 101107, Mar. 1985.

[8] J. S. Petrofsky and C. A. Phillips, "Electrical stimulation under computer control," in Proc. Conf. Frontiers Eng. Comp. Health Care, Columbus, $\mathrm{OH}, 1983$, paper 9.6 .

[9] T. Bajd, A. Kralj, R. Turk, H. Benko, and J. Sěga, "The use of a four-channel electrical stimulator as an ambulatory aid for paraplegic patients,"' Phys. Ther., vol. 63, no. 7, pp. 1116-1120, July 1983

[10] B. Khawam, R. McGovern, and E. B. Marsolais, "Paraplegic subjects' experience in an FNS research program," in Proc. RESNA IOth Ann. Conf., San Jose, CA, June 19-23, 1987, pp. 588-590.

[11] J. Campbell, "Efficiency of volitional versus electrically evoked knee extension excercise," in Proc. RESNA IOth Ann. Conf., San Jose, CA, June 19-23, 1987, pp. 648-650.

[12] S. R. Collins and R. M. Glaser, "Comparison of aerobic, metabolism and cardiopulmonary responses for electrically induced and voluntary exercise," in Proc. RESNA 8th Ann. Conf., Memphis, TN, June 24-28, 1985, pp. 391-393.

[13] G. M. Davis, F. J. Servedio, R. M. Glaser, S. R. Collins, S. C. Gupta, and A. G. Suryaprasad, "Hemodynamic responses during electrically-induced leg and voluntary arm crank exercise in lowerlimb disabled males," in Proc. RESNA lOth Ann. Conf., San Jose, CA, June 19-23, 1987, pp. 591-593.

[14] J. T. Mortimer, "Motor prostheses," in Handhood of PhysiologyThe Nervous System II, Bethesda, MD: American Physiological Society, 1981, ch 5, pp. 155-187.

[15] P. H. Peckham, "Electrical excitation of skeletal muscle: Alterations in force, fatigue, and metabolic properties,' $\mathrm{Ph} . \mathrm{D}$. thesis, Case Western Reserve Univ., Cleveland, OH, Aug. 1972.

[16] J. S. Petrofsky and C. A. Phillips, "Microprocessor controlled stimulation in paralyzed muscle," IEEE NAECON Record, pp. 198$210,1979$.

[17] P. E. Crago, J. T. Mortimer, and P. H. Peckham, "Closed-loop control of force during electrical stimulation of muscle," IEEE Trans. Biomed. Eng., vol. BME-27, pp. 306-312, June 1980.

[18] W. K. Durfee, "Task control with an electrically stimulated antagonist muscle pair," Ph.D. thesis, Cambridge, MA: M.I.T., June 1985 .

[19] W. K. Durfee, "Task-based methods for evaluating electrically stimulated antagonist muscle controllers," IEEE Trans. Biomed. Eng., vol. 36, pp. 309-321, Mar. 1989.

[20] P. H. Gorman and J. T. Mortimer, "The effect of stimulus parameters on the recruitment characteristics of direct nerve stimulation," IEEE Trans. Biomed. Eng., vol. BME-30, pp. 407-414, July 1983

[21] D. R. McNeal, L. L. Baker, and J. T. Symons, "Recruitment data for nerve cuff electrodes: Implications for design of implantable stimulators," IEEE Trans. Biomed. Eng., vol. 36, pp. 301-308, Mar. 1989.

[22] J. S. Petrofsky, "Control of the recruitment and firing frequencies of motor units in electrically stimulated muscles in the cat," Med. Biol. Eng. Comput., vol. 16, pp. 302-308, May 1978

[23] R. Douglas, P. F. Larson, R. D'Ambrosia, and R. E. McCall, "The LSU reciprocation-gait orthosis," Orthoped., vol. 6, pp. 834-839, July 1983.

[24] F. E. Zajac, "Muscle and tendon: Properties, models, scaling and application to biomechanics and motor control," in CRC Critical Reviews in Biomedical Engineering, J. R. Bourne, Ed. Boca Raton, FL: CRC Press, 1989, vol. 17, pp. 359-411.

[25] F. E. Zajac and M. E. Gordon, "Determining muscle's torce and action in multi-articular movement," in Exercise and Sport Science Reviews, K. Pandolf, Ed. Baltimore, MD: Williams \& Wilkins, 1989, vol. 17, pp. 187-230.

[26] S. V. Fisher and G. Gullickson, Jr. "Energy cost of ambulation in health and disability: A literature review," Arch. Phys. Med. Reh abil., vol. 59, pp. 124-133, Mar. 1978.

[27] R. L. Waters and B. R. Lunsford, "Energy cost of paraplegic locomotion,"' J. Bone Joint Surg., vol. 67-A, no. 8, pp. 1245-1250, Oct. 1985
[28] H. J. Chizeck, R. Lalonde, C. W. Chang, J. A. Rosenthal, and E. B. Marsolais, "Performance of a closed-loop controller for electrically-stimulated standing in paralyzed patients, " in Proc. RESNA 8th Ann. Conf., Memphis, TN, June 24-28, 1985, pp. 231-233.

[29] E. Simon, P. Muccio, H. Chizeck, J. Mansour, J. Pereira, and E. B. Marsoloais. "The feasibility of using strain measurements in an ankle-foot orthosis as a feedback signal for closed-loop FNS gait," in Proc. RESNA IOth Ann. Conf., San Jose, CA, June 19-23, 1987, pp. $600-602$.

[30] G. Khang, "Paraplegic standing controlled by functional neuromuscular stimulation: Computer model, control-system design, and simulation studies," Ph.D. thesis, Stanford, CA: Stanford University, June 1988 .

[31] T. Bajd and L. Vodovnik, "Pendulum testing of spasticity," Biomed. Eng., vol. 6, no. 9, pp. 9-16, 1984

[32] G. R. Cybulski, R. D. Penn, and R. J. Jaeger, "Lower extremity functional neuromuscular stimulation in cases of spinal cord injury," Neurosurg, vol. 15, no. 1, pp. 132-146, July 1984.

[33] C. Van den Honert and J. T. Mortimer, "A technique for collision block of peripheral nerve: Single stimulus analysis," IEEE Trans. Biomed. Eng., vol. BME-28, pp. 373-378, May 1981.

[34] A. Kralj, T. Bajd, R. Turk, and H. Benko, "Posture switching for prolonging functional electrical stimulation standing in paraplegic patients," Paraplegia, vol. 24, pp. 221-230, 1986.

[35] J. S. Petrofsky, "Sequential motor unit stimulation through peripheral motor nerves in the cat," Med. Biol. Eng. Comput., vol. 17, pp. 87-93, Jan. 1979

[36] G. T. Yamaguchi, "Feasibility and conceptual design of functional neuromuscular stimulation systems for the restoration of natural gait to paraplegics based on dynamic musculoskeletal models," Ph.D. thesis, Stanford, CA: Stanford Univ., Aug. 1989.

[37] M. L. Audu and D. T. Davy, "The influence of muscle model complexity in musculoskeletal motion modeling," J. Biomech. Eng., vol. 107, pp. 147-157, May 1985.

[38] D. T. Davy and M. L. Audu, "A dynamic optimization technique for predicting forces in the swing phase of gait," J. Biomechan. vol. 20 , no. 2 , pp. $187-201$, Feb. 1987.

[39] H. Hemami, V. C. Jaswa, and R. B. McGhee, "Some alternative formations of manipulator dynamics for computer simulation studies," in Proc. 13th Allerton Conf. Circuit Theory, Univ. Illinois, Chicago, Oct. 1975

[40] F. E. Zajac, P. J. Stevenson, and E. L. Topp, "A dimensionless musculotendon actuator model for use in computer simulations of body coordination: Static properties," in Proc. N. Amer. Congress Biomechan., Montreal, Quebec, Aug. 25-27, 1986, pp. 245-246.

[41] F. E. Zajac, P. J. Stevenson, and E. L. Topp, "A dimensionless musculotendon model," in Proc. 8th Ann. Conf. IEEE Eng. Med. Biol. Soc., Ft. Worth, TX, 1986, pp. 601-604.

[42] R. A. Brand, R. D. Crowninshield, C. E. Wittstock, D. R. Pedersen, C. R. Clark, and F. M. Van Krieken, "A model of lower extremity muscular anatomy," J. Biomech. Eng., vol. 104, pp. 304310 , Nov. 1982

[43] M. G. Hoy, F. E. Zajac, and M. E. Gordon, “A musculoskeletal model of the human lower extremity: The effect of muscle, tendon, and moment arm on the moment-angle relationship of musculotendon actuators at the hip, knee, and ankle," J. Biomech., vol. 23, no. 2 , pp. 157-169, 1990.

[44] G. T. Yamaguchi and F. E. Zajac, "A planar model of the knee joint to characterize the knee extensor mechanism," J. Biomechan., vol. 22 , no. 1, pp. 1-10, Jan. 1989.

[45] R. A. Mann and J. L. Hagy, "The function of the toes in walking, jogging and running," Clin. Orthop. Rel. Res., no. 142, pp. 2429, July-Aug. 1979.

[46] D. E. Kirk, Optimal Control Theory. An Introduction. Englewood Cliffs, NJ: Prentice-Hall, 1970.

[47] R E Larson, and J L Casti, Principles of Dynamic Programming. Part I. Basic Analytic and Computational Methods. New York, NY: Marcel Dekker, 1978.

[48] T. R. Kane and D. A. Levinson, Dynamics: Theory and Applications. New York: McGraw-Hill, 1985.

[49] R. D. Crowninshield and R. A. Brand, "A physiologically based criterion of muscle force prediction in locomotion," J. Biomechan. vol. 14, no. 11, pp. 793-801, Nov. 1981.

[50] D. A. Winter, The Biomechanics and Motor Control of Human Gait. Waterloo, Ontario (Canada): University of Waterloo Press, 1987.

[51] V. T. Inman, H. J. Ralston, and F. Todd, Human Walking. Baltimore, MD: Williams \& Wilkins, 1981 
[51a] L. M. Nashner and G. McCollum, "The organization of human postural movements: A formal basis and experimental synthesis," $\mathrm{Be}$ havioral Brain Sci., vol. 8, pp. 135-172, 1985.

[52] J. Perry, "Normal and pathological gait," in American Academy of Orthopaedic Surgeons (AAOS) Atlas of Orthotics, 2nd Ed. St. Louis, MO: C. V. Mosby, 1985, pp. 76-111.

[53] L. M. Nasher, "Fixed patterns of rapid postural responses among leg muscles during stance,"'Exp. Brain Res., vol. 30, pp. 13-24, 1977.

[54] G. Khang and F. E. Zajac, "Paraplegic standing controlled by functional neuromuscular stimulation: Part ll-Computer simulation studies," IEEE Trans. Biomed. Eng., vol. 36, pp. 885-894, Sept. 1989.

[55] — , "Paraplegic standing controlled by functional neuromuscular stimulation: Part I-Computer model and control-system design," IEEE Trans. Biomed. Eng., vol. 36, pp. 873-884, Sept. 1989.

[56] Univ. California, Berkeley, "The patterns of muscular activity in the lower extremity during walking," in Prosthetic Division Report, Series 11, Issue 25, 1953.

[57] A. G. Patriarco, R. W. Mann, S. R. Simon, and J. M. Mansour, "An evaluation of the approaches of optimization models in the prediction of muscle forces during human gait," J. Biomech., vol. 14, no. 8, pp. 513-525, Aug. 1981.

[58] K. Lyons, J. Perry, J. K. Gronley, L. Barnes, and D. Antonelli, "Timing and relative intensity of hip extensor and abductor muscle action during level and stair ambulation-An EMG study," J. Amer. Phys. Therapy Assn., vol. 63, no. 10, pp. 1597-1605, Oct. 1983.

[59] R. Shiavi, "Electromyographic patterns in adult locomotion: A comprehensive review," J. Rehab. $R \& D$, , vol. 22 , no. 3, pp. 8598, July 1985 [contains EMG recordings from Mann and Inman (1964), Sullivan (1966), Perry (1974), and Winter (1984)].

[60] H. Elftman, "Biomechanics of muscle-With particular application to studies of gait," J. Biomech., vol. 48-A, no. 2, pp. 363-377, 1966.

[61] A. L. Hof, B. A. Geelen, and Jw. Van den Berg, "Calf muscle moment, work and efficiency in level walking: Role of series elasticity," J. Biomech, vol. 16, no. 7, pp. 523-537, 1983.

[62] P. E. Muccio and E. B. Marsolais, "Experience with ankle-foot orthoses and paraplegic subjects using functional neuromuscular stimulation," in Proc. RESNA 10th Ann. Conf., San Jose, CA, June 19-23, 1987, pp. 613-615.
[63] J. L. Stein, "Design issues in the stance phase control of above knee protheses," Ph.D. thesis, Cambridge, MA: M.I.T., 1983.

[64] M.-S. Ju and J. M. Mansour, "Stimulation of the double limb support phase of human gait," J. Biomech. Eng., vol. 110, pp. 223229, Aug. 1988.

[65] R. B. Stein, "What muscle variable(s) does the nervous system control in limb movement?"' Behavior. Brain Sci., vol. 5, pp. 535-577, 1982.

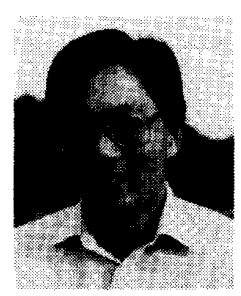

Gary T. Yamaguchi was born in Seattle, WA, in 1956. He received the B.S. degree and the A.B. degree from the California Institute of Technology and Occidental College in 1979, the S.M. degree in mechanical engineering from the Massachusetts Institute of Technology, Cambridge, in 1981, and the Ph.D. degree in mechanical engineering from Stanford University, Stanford, CA, in 1989 .

From 1981 to 1984 he was employed as a systems integration engineer with the magnetic fusion energy division at the Lawrence Livermore National Laboratory, Livermore, $\mathrm{CA}$, followed by the position of biomedical engineer at the R\&D Center, Palo Alto Veterans Affairs Medical Center, Palo Alto, CA, from 1985 to 1989. Since the summer of 1989 he has been an Assistant Professor of Biomedical Engineering at the Arizona State University, Tempe, AZ. His teaching and reseach interests include biomechanical modeling, analysis, and control, and rehabilitation engineering design.

Dr. Yamaguchi is a member of Sigma Pi Sigma.

Felix E. Zajac (M'70), for a photograph and biography, see p. 766 of the August 1990 issue of this Transactions. 